\title{
A New Two-Stage Fuzzy Inference System-Based Approach to Prioritize Failures in Failure Mode and Effect Analysis
}

\author{
Tze Ling Jee, Kai Meng Tay, Member, IEEE, and Chee Peng Lim
}

\begin{abstract}
This paper presents a new Fuzzy Inference System (FIS)-based Risk Priority Number (RPN) model for the prioritization of failures in Failure Mode and Effect Analysis (FMEA). In FMEA, the monotonicity property of the RPN scores is important. To maintain the monotonicity property of an FIS-based RPN model, a complete and monotonically-ordered fuzzy rule base is necessary. However, it is impractical to gather all (potentially a large number of) fuzzy rules from FMEA users. In this paper, we introduce a new two-stage approach to reduce the number of fuzzy rules that needs to be gathered, and to satisfy the monotonicity property. In stage-1, a Genetic Algorithm (GA) is used to search for a small set of fuzzy rules to be gathered from FMEA users. In stage-2, the remaining fuzzy rules are deduced approximately by a monotonicity-preserving similarity reasoning scheme. The monotonicity property is exploited as additional qualitative information for constructing the FIS-based RPN model. To assess the effectiveness of the proposed approach, a real case study with information collected from a semiconductor manufacturing plant is conducted. The outcomes indicate that the proposed approach is effective in developing an FIS-based RPN model with only a small set of fuzzy rules, which is able to satisfy the monotonicity property for prioritization of failures in FMEA.
\end{abstract}

Index Terms-Failure mode and effect analysis, fuzzy inference system, similarity reasoning, monotonicity property, fuzzy rule reduction.

\section{ABBREVIATIONS \& ACRONYMS}

AARS Approximate Analogical Reasoning Schema

\section{Detection}

FCBGA Flip Chip Ball Grid Array

FIS Fuzzy Inference System

FMEA Failure Mode and Effect Analysis

FRI Fuzzy Rule Interpolation

FRPN fuzzy RPN

GA Genetic Algorithm

O Occurrence

Manuscript received August 19, 2013; revised July 03, 2014; accepted November 19, 2014. Date of publication May 08, 2015; date of current version August 28, 2015. This work was supported in part by the FRGS grant (i.e., FRGS/ICT02(01)/997/2013(38)) and in part by the RACE grant (i.e., RACE/F2/TK/UNIMAS/5). Associate Editor. C. Smidts.

T. L. Jee and K. M. Tay are with the Faculty of Engineering, Universiti Malaysia Sarawak, Kota Samarahan, Sarawak, Malaysia (e-mail: kmtay@feng. unimas.my; tkaimeng@yahoo.com).

C. P. Lim is with the Centre for Intelligent Systems Research, Deakin University, Australia.

Digital Object Identifier 10.1109/TR.2015.2420300

$\begin{array}{ll}\text { MF } & \text { membership function } \\ \text { NLP } & \text { Non-Linear Programming } \\ \text { RPN } & \text { Risk Priority Number } \\ S & \text { Severity } \\ S-1 F R s & \text { Stage 1 Fuzzy Rules } \\ S-2 F R s & \text { Stage 2 Fuzzy Rules } \\ \text { SQP } & \text { Sequential Quadratic Programming } \\ \text { SR } & \text { Similarity Reasoning } \\ & \\ & \quad \text { A membership function for } x \in[S, O, D], \text { where } \\ \mu_{x}^{n_{x}}(x) & n_{x}=1,2,3, \ldots, m_{x} \\ A_{x}^{n_{x}} & \text { A linguistic term for } x \in[S, O, D], \text { where } \\ & n_{x}=1,2,3, \ldots, m_{x} \\ m_{x} & \text { Number of membership function for } x \in[S, O, D] \\ p_{x} & p_{x} \in\left[1,2,3, \ldots, m_{x}-1\right] \\ x & \text { An input for RPN model, } x \in[S, O, D]\end{array}$

\section{INTRODUCTION}

$\mathbf{F}$ AILURE mode and effect analysis (FMEA) is a popular reliability analysis tool that is used to evaluate the risks associated with potential failure modes of a complex system or process [1]-[3]. In FMEA, the risk of a failure mode is determined by a Risk Priority Number (RPN) [1], i.e., $R P N=$ $f(S, O, D)$ whereby three risk factors, i.e., Severity $(S), O c$ currence $(O)$, and Detection $(D)$, act as the inputs, and an RPN score is produced as the output. In this aspect, the fuzzy RPN model has been successfully applied to a variety of domains, which include maritime [3], fishing vessel [4], manufacturing [5], power generation [6], product development [7], and agriculture [8]. The focus of this paper is on the use of the Fuzzy Inference System (FIS) in FMEA, i.e., the FIS-based RPN model [9]. The advantages of using the FIS-based RPN model, as compared with the conventional RPN model, are well-established, viz., (i) the FIS-based model allows modeling of nonlinear relationships between RPN and risk factors [9]; (ii) FIS is a solution for the attribute scales, which can be qualitative, instead of quantitative [9]; (iii) FIS is able to incorporate human knowledge, whereby information can be described with vague and imprecise linguistic statements [10]; and (iv) FIS avoids the scenario whereby two or more sets of $S, O$ and $D$ settings with 